\title{
Aspectos fundamentales de la flotación de hematita usando biosurfactante
}

\author{
Carlos Alberto Castañeda Olivera \\ Departamento de Ingeniería Química y de Materiales, Pontificia Universidad Católica de Rio de Janeiro, Rua \\ Marquês de São Vicente, 225 - Gávea - Rio de Janeiro - CEP: 22453-900, Brasil
}

Recibido 3 de agosto. Revisado 8 de diciembre. Aprobado 17 de diciembre 2019.

DOI: https://doi.org/10.33017/RevECIPeru2019.0008/

\section{Resumen}

El uso de biosurfactantes como reactivos de flotación representa un gran potencial tecnológico y aceptabilidad ambiental en la industria mineral. Siendo así, esta investigación estudia los aspectos fundamentales de la flotación de hematita utilizando el biosurfactante (BS) producido por Rhodococcus erythropolis. El BS fue caracterizado por análisis de bioquímica y mediciones de tensión superficial, mientras que la interacción hematita-BS fue verificada a través de mediciones de potencial zeta, medidas de ángulo de contacto, experimentos de adsorción y espectros FTIR. Los testes de microflotación fueron realizados en tubo de Hallimond modificado y el máximo valor de flotabilidad $(99,87 \%)$ fue encontrado en $\mathrm{pH} 3$ con una concentración de BS de $100 \mathrm{mg} / \mathrm{L}$ para un tiempo de flotación de $1 \mathrm{~min}$. Los datos de la flotabilidad de hematita fueron analizados por regresión polinómica para obtener una función representativa de la microflotación, así como también sus respectivas superficies de respuesta. Finalmente, los resultados mostraron que o biosurfactante producido por Rhodococcus erythropolis puede actuar como un bioreactivo colector en la flotación de hematita.

Descriptores: biosurfactante, flotación, hematita, Rhodococcus erythropolis.

\section{Abstract}

The use of biosurfactants as flotation reagents represents a great technological potential and environmental acceptability in the mineral industry. Thus, this research studies the fundamental aspects of hematite flotation using the biosurfactant (BS) produced by Rhodococcus erythropolis. The BS was characterized by biochemical analysis and surface tension measurements, while the hematite-BS interaction was verified through measurements of zeta potential, contact angle measurements, adsorption experiments and FTIR spectra. The microflotation tests were performed in modified Hallimond tube and the maximum floatability value $(99.87 \%)$ was found in $\mathrm{pH} 3$ with a BS concentration of $100 \mathrm{mg} / \mathrm{L}$ for a flotation time of $1 \mathrm{~min}$. The hematite floatability data were analyzed by polynomial regression to obtain a representative function of the microflotation, as well as their respective response surfaces. Finally, the results showed that the biosurfactant produced by Rhodococcus erythropolis can act as a collector bioreagent in the hematite flotation.

Keywords: biosurfactant, flotation, hematite, Rhodococcus erythropolis.

\section{Introducción}

La revisión de la literatura ha demostrado que algunos microorganismos pueden producir compuestos tensioactivos que pueden variar en su estructura química y tamaño. Estos biosurfactantes, se consideran en la actualidad como sustitutos potenciales de los surfactantes convencionales (producidos químicamente) debido a su menor toxicidad, mejor compatibilidad ambiental, biodegradabilidad y efectividad en un amplio rango de temperaturas y $\mathrm{pH}[1]$.

Los biosurfactantes son compuestos bioquímicos que poseen una estructura común composta de una porción lipofílica y una porción hidrofílica [2]. Tienen 
las mismas características de los surfactantes químicos, por lo que se pueden aplicar en las industrias de tratamiento y procesamiento de metales [3].

Se ha descrito que el género Rhodococcus comprende importantes microrganismos productores de biosurfactantes, que forman compuestos tensioactivos derivados de trehalosa, especialmente utilización de fuentes de carbono [4]. La bacteria Rhodococcus erythropolis es un microorganismo Gram-positivo no patógeno que se encuentra en una amplia variedad de fuentes, incluyendo suelos, rocas, aguas subterráneas y sedimentos marinos [5]. Esta bacteria ha sido ampliamente utilizada para la bioremediación de aguas y solos contaminados con petróleo [6,7], así como también, como reactivo colector de hematita, usando su biomasa [8]. Por lo tanto, el presente trabajo estudia la flotación de hematita utilizando el biosurfactante producido por Rhodococcus erythropolis.

\section{Metodología}

\subsection{Muestra mineral}

El mineral fue obtenido de la ciudad Araçuaí en Minas Gerais - Brasil. El grado de pureza de la muestra mineral fue de $95,63 \% \mathrm{Fe}_{2} \mathrm{O}_{3}$. La muestra mineral fue triturada y molida en seco, luego fue tamizada en húmedo para obtener las fracciones granulométricas deseadas (Tabla 1).

Tabla 1: Ensayos y fracciones granulométricas.

\begin{tabular}{|c|c|}
\hline Ensayos & Fracciones \\
\hline Potencial Zeta & $<38 \mu \mathrm{m}$ \\
\hline Ángulo de contacto & $1 \times 1 \times 2 \mathrm{~cm}$ \\
\hline Adsorción & $(-53+38 \mu \mathrm{m})$ \\
\hline Microflotación & $(-106+75 \mu \mathrm{m})$ \\
\hline
\end{tabular}

2.2 Medio de cultivo, crecimiento del microorganismo y extracción del biosurfactante

La cepa bacteriana $R$. erythropolis fue obtenida de la Colección Brasileira de Microorganismos de Ambiente e Industria - CBMAI - UNICAMP. El microorganismo fue cultivado usando el medio de cultura TSB consistente de extracto de caseína (17 $\mathrm{g} / \mathrm{L})$, extracto de harina de soya $(3 \mathrm{~g} / \mathrm{L})$, Clorato de sodio (5 g/L), Fosfato dipotásico $(2,5 \mathrm{~g} / \mathrm{L})$ y glucosa $(2,5 \mathrm{~g} / \mathrm{L})$. El crecimiento del microorganismo fue realizado en medio líquido usando frascos Erlenmeyer de $500 \mathrm{~mL}$ debidamente esterilizados, para luego ser llevados a un shaker rotativo a una temperatura de $28^{\circ} \mathrm{C}$ por $144 \mathrm{~h}$. Las células obtenidas fueron centrifugadas y lavadas dos veces con agua desionizada. La extracción del biosurfactante siguió el procedimiento de [3].

\subsection{Medidas de potencial zeta}

Las medidas de potencial zeta se realizaron para la hematita y su interacción hematita-biosurfactante, usando el equipamiento Zeta Meter 4.0+ System. Fueron preparadas soluciones con concentraciones de $50 \mathrm{mg} / \mathrm{L}$. Para mantener la fuerza iónica de la solución se empleó $\mathrm{NaCl} 0,001 \mathrm{~mol} / \mathrm{L}$. El pH de la solución fue ajustado con alícuotas de $\mathrm{HCl}$ y $\mathrm{NaOH}$.

\subsection{Medidas de ángulo de contacto}

Las medidas de ángulo de contacto fueron realizadas utilizando un Goniómetro modelo RaméHart de la COPPE/UFRJ. La sección de mineral fue moldada con resina epoxi y posteriormente, la muestra fue lijada y pulida hasta llegar a $1 \mu \mathrm{m}$.

\subsection{Experimentos de adsorción}

Los experimentos de adsorción se realizaron con $0,25 \mathrm{~g}$ de muestra mineral. Las soluciones se prepararon con diferentes concentraciones de biosurfactante y se acondicionaron durante 5 minutos después de ajustarse el pH deseado. La cantidad de biosurfactante adsorbido en la superficie mineral se determinó mediante mediciones de absorbancia, utilizando el espectrofotómetro Shimadatzu UV-1800 y su interacción se registró en el espectrómetro Nicolet6700 FTIR.

\subsection{Análisis en el espectrofotómetro de infrarrojo con transformada de Fourier (IRTF)}

Los análisis de infrarrojo fueron realizados utilizando un espectrofotómetro Nicolet 6700 . El polvo resultante de las muestras secas fue misturado con una matriz de bromato de potasio $(\mathrm{KBr})$ en una relación de $1 \%(\mathrm{w} / \mathrm{w})$. Estos espectros fueron usados para comparar los grupos funcionales presentes en el biosurfactante y en la muestra de hematita, antes y después de la interacción con el biosurfactante.

\subsection{Testes de microflotación}

Los testes de microflotación se llevaron a cabo en un tubo Hallimond modificado con $160 \mathrm{~mL}$ de solución, utilizándose $1 \mathrm{~g}$ de mineral. El mineral fue acondicionamiento en el $\mathrm{pH}$ deseado a una agitación constante por $3 \mathrm{~min}$, posteriormente fue 
realizada la flotación mineral con una alimentación de aire equivalente a $25 \mathrm{~mL} / \mathrm{min}$, durante 1 minuto.

El material flotado y no-flotado fue lavado, secado y pesado. La flotabilidad de los minerales fue calculada como la relación de masa entre el material flotado y la masa inicial del material.

Los resultados de microflotación fueron evaluados mediante regresión polinomial usando el software MatLab R2012b y como resultado fueron obtenidos gráficos de superficies de respuesta, así como también una función polinomial representativa de la microflotación mineral.

El modelo fue analizado mediante el criterio del valor-p (probabilidad), usando 0,05 como el límite para la significancia estadística.

\section{Resultados}

\subsection{Biosurfactante}

El biosurfactante extraído es predominantemente glicolípido ligado a células conteniendo trehalosa [9]. Los surfactantes como los trehalolípidos asociados a células Rhodococcus pueden ser eficazmente aislados por la extracción con solvente orgánico [10]. Dentro de la gran variedad de solventes orgánicos que han sido utilizados, aisladamente o en combinación de ellos, tenemos: metanol, etanol, éter dietílico, pentano, acetona, cloroformo y diclorometano [11], así como también el éter metil-butil terciario (MTBE) por ser un solvente menos toxico y barato [9].

\subsection{Medidas de potencial zeta}

En la Figura 1, son mostrado los resultados de las curvas de potencial zeta de la hematita, tanto antes como después de la interacción con el biosurfactante.

Las curvas de potencial zeta indicaron que las partículas de hematita presentaron un valor de punto isoeléctrico (PIE) alrededor del pH 5,2. Ese valor es similar a los valores encontrados por [5,8,12], donde sus PIEs fueron en el rango de $\mathrm{pH} 5,0-6,0$. Después de la interacción con el biosurfactante, se observó mudanzas en el perfil de la curva de potencial zeta y su PIE mudó para el valor de $\mathrm{pH} \mathrm{4,4}$. Esa mudanza en el valor de PIE puede ser debido a las interacciones surfactantes ocurridas en la superficie mineral [5,13-15].

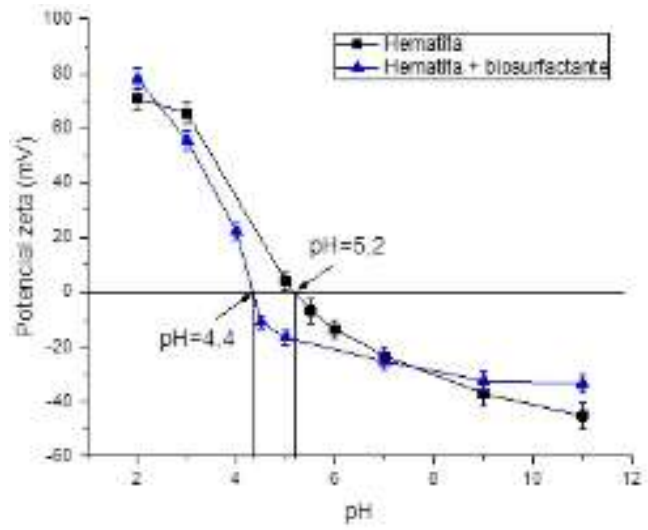

Figura 1: Curvas de Potencial zeta de la hematita en función del $\mathrm{pH}$ (electrólito indiferente: $\mathrm{NaCl} \mathrm{10^{-2 }}$ $\mathrm{mol} / \mathrm{L}$ )

\subsection{Medidas de ángulo de contacto}

Antes de la interacción con el biosurfactante, la superficie de hematita presentó un bajo carácter hidrofóbico, cuyo valor de ángulo de contacto fue de $31,5^{\circ} \pm 8,65^{\circ}$. Sin embargo, después de la interacción, la superficie de hematita tuvo un aumento del carácter hidrofóbico. La Figura 2 muestra las medidas de ángulo de contacto de la hematita realizadas en función del $\mathrm{pH}$. Observase que las mayores medidas de ángulo de contacto fueron alcanzadas en el rango de $\mathrm{pH} 3$ hasta 4, con valores alrededor de $56^{\circ}$. Ese resultado fue comparado con el trabajo de [16], donde estudiaron la interacción de la hematita con la biomasa de Rhodococcus opacus. En la superficie de hematita fue verificado un aumento del carácter hidrofóbico, con valores de $31^{\circ}-36^{\circ}$ en el rango de $\mathrm{pH} 3$ hasta 5.

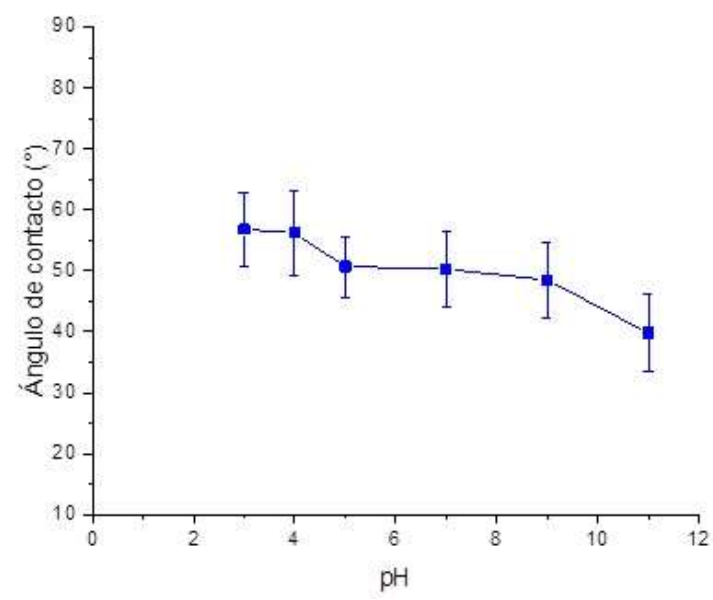

Figura 2: Medidas de ángulo de contacto de la superficie de hematita después de la interacción con el biosurfactante (concentración del biosurfactante: 100 $\mathrm{mg} / \mathrm{L}$ ) 


\subsection{Experimentos de adsorción}

La Figura 3 muestra los resultados de adsorción del biosurfactante sobre la superficie de hematita. Los mismos muestran que en todas las concentraciones utilizadas, la mayor adsorción se logró en el rango de $\mathrm{pH}$ ácido, principalmente a $\mathrm{pH} 3$. También se observó que cuanto mayor es la concentración de biosurfactante, mayor es la capacidad de adsorción. Por lo tanto, para la concentración de biosurfactante de $200 \mathrm{mg} / \mathrm{L}$, la cantidad máxima de biosurfactante adsorbida sobre superficie de hematita fue de 11.62 mg por gramo de mineral.

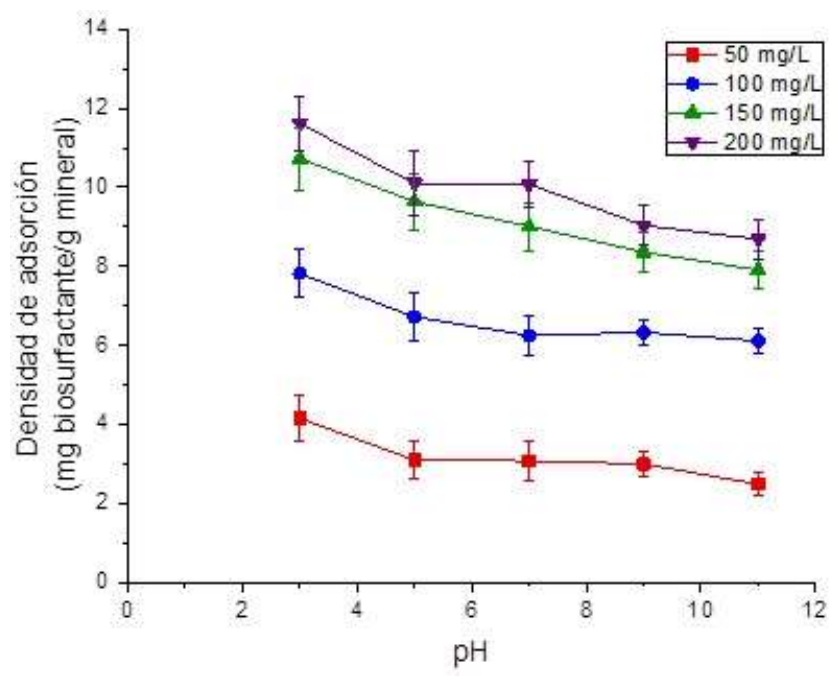

Figura 3: Adsorción del biosurfactante sobre la superficie de hematita

Según [17] y [18], la alta adsorción en los valores de $\mathrm{pH}$ ácido se relaciona con fuerzas electrostáticas atractivas entre las cargas negativas del biosurfactante y la superficie positiva de la hematita, mientras que la disminución de la adsorción en los valores de $\mathrm{pH}$ alcalinos se relaciona con la repulsión electrostática entre las cargas negativas del biosurfactante y la superficie de hematita.

\subsection{Análisis de infrarrojo con transformada de Fourier (IRTF)}

La Figura 4, presenta el espectrograma de infrarrojo del biosurfactante mostrando sus principales bandas. Esas bandas fueron interpretadas a partir de los resultados encontrados en la literatura [5,8,19-21]. Siendo así, la banda 3417,64 $\mathrm{cm}^{-1}$ es atribuida a las vibraciones de estiramiento de grupos $\mathrm{OH}$ y $\mathrm{NH}$; las bandas en regiones entre 3000-2800 son características del estiramiento $\mathrm{C}-\mathrm{H}$ de los grupos $\mathrm{CH}_{2}$ y $\mathrm{CH}_{3}$; la banda $1634,34 \mathrm{~cm}^{-1}$ es característica del estiramiento $\mathrm{C}=\mathrm{O}$ de la amida I en la proteína; las bandas entre 1500-1300 cm-1 corresponden a las vibraciones de flexión de los grupos $\mathrm{CH}_{2}$ y $\mathrm{CH}_{3}$; la banda $1080,28 \mathrm{~cm}^{-1}$ es característica del estiramiento asimétrico de grupos fosfato en ácidos teicoicos y a los modos complejos de vibración de los polisacáridos; la banda 1048,68 $\mathrm{cm}^{-1}$ es atribuida al estiramiento del grupo alcohólico primario $\left(\mathrm{CH}_{2} \mathrm{OH}\right)$ y la banda $543,04 \mathrm{~cm}^{-1}$ puede ser atribuida a las vibraciones de balance del grupo $\mathrm{CH}_{2}$.

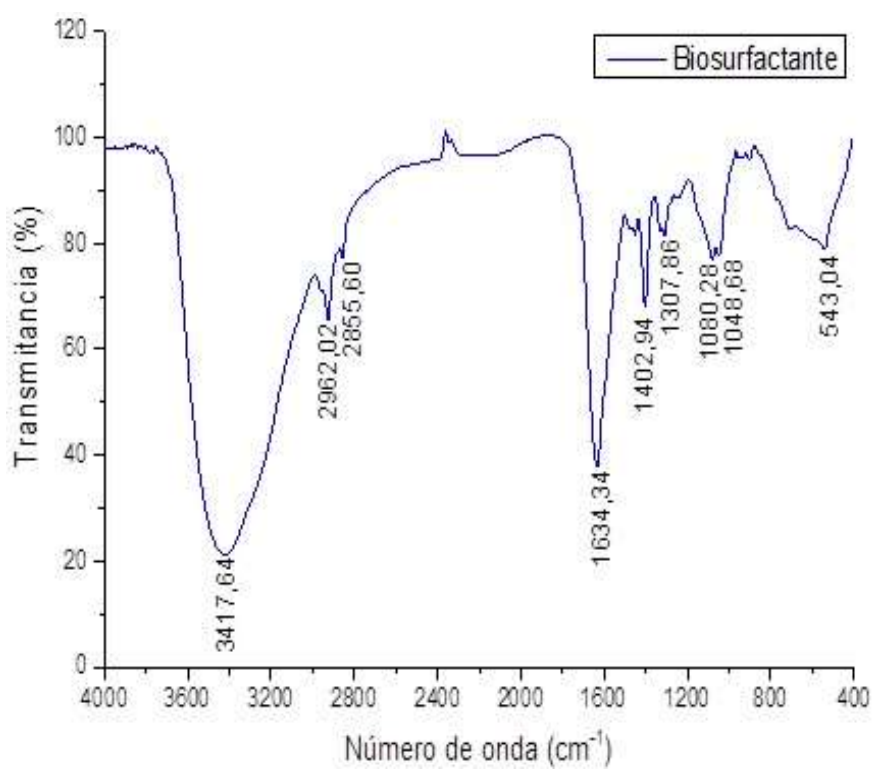

Figura 4: Espectrograma de Infrarrojo del biosurfactante

El espectrograma de infrarrojo de la hematita y interacción hematita-biosurfactante es mostrado en la Figura 5. Antes de la interacción, la superficie de hematita mostró grupos hidroxilo $(\mathrm{OH})$ y vibraciones de estiramiento (Fe-O). Después de la interacción, se observó que la superficie de hematita contiene seis grupos característicos del biosurfactante que fueron dislocados en diferentes direcciones, entre ellos: las vibraciones de estiramiento de grupos $\mathrm{OH}$ y NH mudaron de $3417,64 \mathrm{~cm}^{-1}$ para $3426,48 \mathrm{~cm}^{-1}$; el estiramiento $\mathrm{C}-\mathrm{H}$ dos grupos $\mathrm{CH}_{2}$ e $\mathrm{CH}_{3}$ mudaron de $2962,02 \mathrm{~cm}^{-1}$ para $2925,98 \mathrm{~cm}^{-1}$; el estiramiento $\mathrm{C}=\mathrm{O}$ de la amida I en la proteína mudó de 1634,34 $\mathrm{cm}^{-1}$ para $1630,63 \mathrm{~cm}^{-1}$; las vibraciones de flexión dos grupos $\mathrm{CH}_{2}$ y $\mathrm{CH}_{3}$ mudaron de 1402,4 y $1307,86 \mathrm{~cm}^{-1}$ para 1403,76 y $1316,52 \mathrm{~cm}^{-1}$, respectivamente $\mathrm{y}$, el estiramiento asimétrico de grupos fosfato mudó de $1080,28 \mathrm{~cm}^{-1}$ para 1080,98 $\mathrm{cm}^{-1}$.

Los resultados de IRTF mostraron que acontecen varios mecanismos de adsorción entre el biosurfactante y la superficie mineral, así como también interacciones químicas entre ellos. 


\subsection{Testes de microflotación}

La Figura 6 muestra los resultados de flotabilidad de los diferentes testes de microflotación. Esos mismos datos experimentales fueron analizados mediante regresión polinómica.

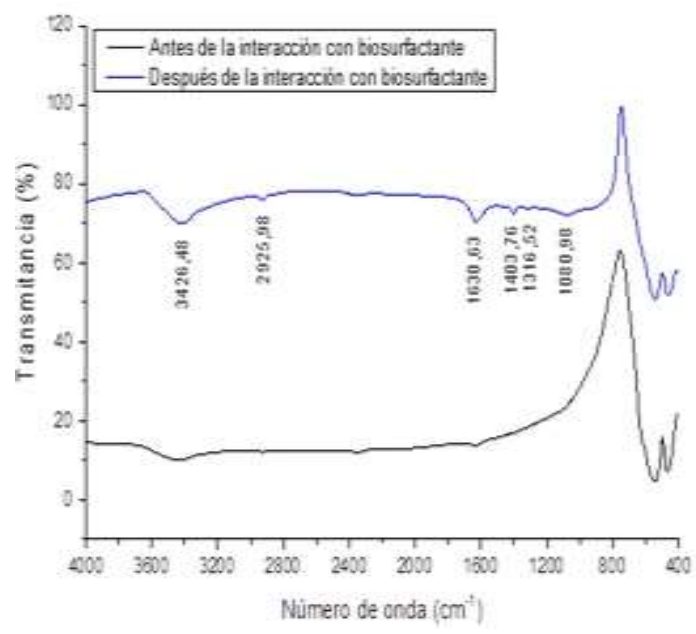

Figura 5: Espectrograma de Infrarrojo de la hematita (antes y después de la interacción con el biosurfactante)

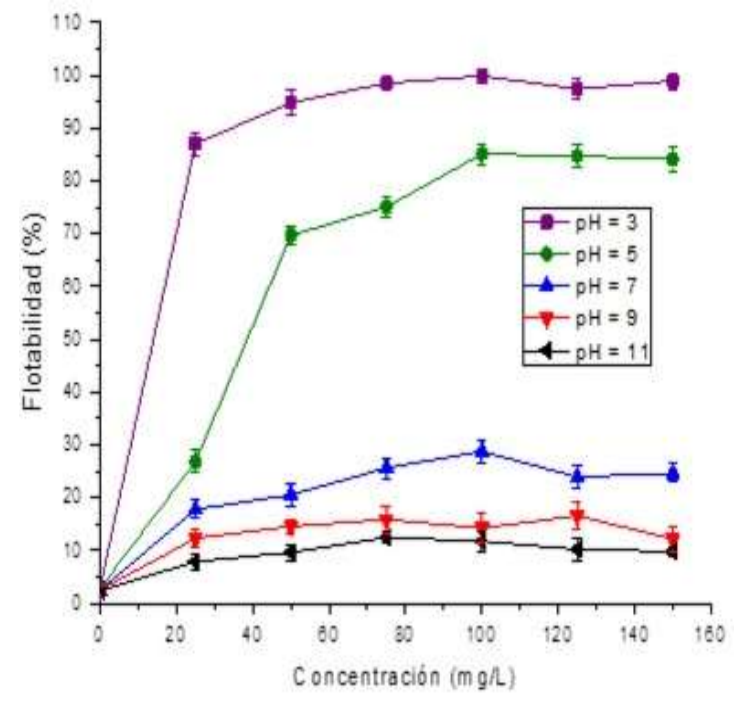

Figura 6: Flotabilidad de la hematita en función de la concentración de biosurfactante

Los resultados mostraron que la hematita tuvo mejor flotabilidad en el $\mathrm{pH}$ ácido, principalmente en el $\mathrm{pH} 3$, con valores de flotabilidad de $99,87 \%$. Ese efecto fue confirmado en las mediciones de ángulo de contacto donde los mejores valores de hidrofobicidad fueron alcanzados en el valor de $\mathrm{pH}$ 3. En ese $\mathrm{pH}$, la interacción del biosurfactante con la superficie de hematita se mostró fuerte, tal como fue confirmado por los estudios de potencial zeta donde se reveló que hubo grande mudanza en el perfil de la curva de hematita después de la interacción con el biosurfactante, provocando el dislocamiento del PIE.

El modelo de regresión polinomial ajustado a partir de los datos de flotabilidad de hematita es una función de orden $4{ }^{\text {ta }}$ para el $\mathrm{pH}$ de la solución y de orden $3^{\text {ra }}$ para la concentración del biosurfactante, con un coeficiente de correlación $\left(R^{2}\right)$ igual a $95,46 \%$. Dicho modelo es mostrado en la Ecuación 1 y sus respectivos valores de los coeficientes son mostrados en la Tabla 2.

$$
f(x, y)=a+b x+c y+d x^{2}+e y^{2}+f x^{3}+g x^{2} \cdot y+h x \cdot y^{2}
$$$$
+i y^{3}+j x^{4}+k x^{3} \cdot y+l x \cdot y^{3}
$$

Dónde: $x=p H$ de la solución, $y=$ Concentración del biosurfactante y las letras a, b, c, d, e, f, g, h, i, j, k, I $=$ Coeficientes del modelo (Tabla 2 ).

Tabla 2: Coeficientes del modelo de regresión polinomial de la microflotación de hematita.

\begin{tabular}{|c|c|}
\hline Coeficientes & Valor \\
\hline a & $-254,13$ \\
\hline b & 203,75 \\
\hline c & 3,603 \\
\hline d & $-53,959$ \\
\hline e & $-0,047844$ \\
\hline f & 5,7515 \\
\hline g & $-0,10018$ \\
\hline h & 0,0062133 \\
\hline i & 0,00013689 \\
\hline j & $-0,21243$ \\
\hline k & 0,0058929 \\
\hline l & $-1,4222 \mathrm{E}-05$ \\
\hline
\end{tabular}

A superficie de respuesta de la microflotación de hematita es presentada en la Figura 7-a y las diferentes zonas del proceso son presentadas en la gráfica de contornos (Figura 7-b). A partir del mismo, podemos apreciar que la mejor zona de microflotación de hematita es en el rango de $\mathrm{pH}$ ácido, usando concentraciones de biosurfactante entre 50 y $150 \mathrm{mg} / \mathrm{L}$.

\section{Conclusiones}

A utilización del biosurfactante producido por la bacteria Rhodococcus erythropolis puede actuar como bioreactivo colector en la flotación de hematita, mostrándose bastante favorable para ser utilizado industrialmente. Tales resultados fueron 
verificados por las mediciones de potencial zeta, medidas de ángulo de contacto, experimentos de adsorción y espectrogramas de infrarrojo.
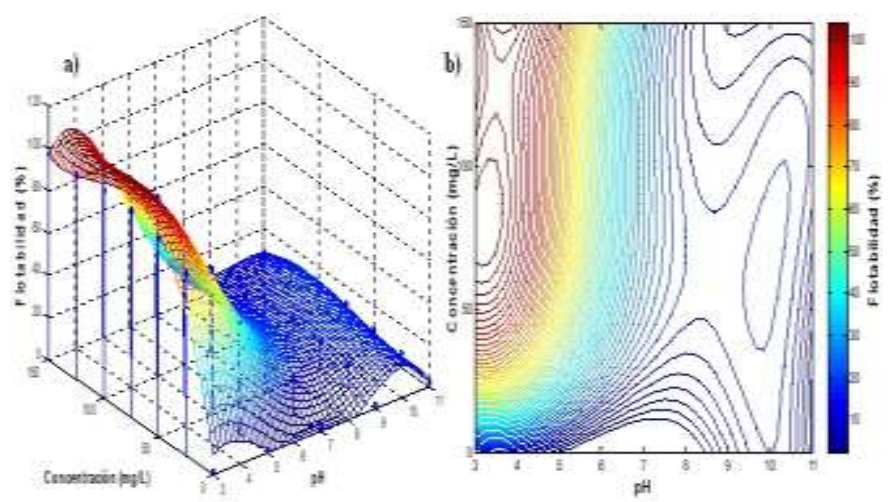

Figura 7: a) Superficie de respuesta $3 D$ de la microflotación de hematita en función de la concentración del biosurfactante y del $\mathrm{pH}$ de la solución; b) Gráfica de contorno $3 D$ de la microflotación de hematita en función de la concentración del biosurfactante y del $\mathrm{pH}$ de la solución.

Los mejores resultados de flotabilidad de hematita fueron encontrados en el $\mathrm{pH} 3$ y en la concentración de biosurfactante de $100 \mathrm{mg} / \mathrm{L}$, con porcentaje de $99,87 \%$.

El modelo de regresión polinomial determinó que a flotabilidad de hematita sigue una función de orden $4^{\text {ta }}$ para el $\mathrm{pH}$ de la solución y de orden $3^{\text {ra }}$ para la concentración del biosurfactante.

\section{Agradecimientos}

Los autores agradecen a CAPES, CNPq y a la PUCRio por el apoyo financiero.

\section{Referencias}

[1] H. Khoshdast et al., Int. Biodeterior. Biodegrad. 65 (2011) 1238.

[2] M. Nitschke and G. M. Pastore, Quím. Nova 25 (2002) 772.
[3] C. A. C. Olivera, Doctoral Thesis, Pontifical Catholic University of Rio de Janeiro, 2018.

[4] G. Retamal-Morales et al., J. Biotechnol. 280 (2018) 38.

[5] C. A. C. Olivera, A. G. Merma, J. G. S. Puelles, and M. L. Torem, Miner. Eng. 106 (2017) 55.

[6] C.-W. Liu and H.-S. Liu, Process Biochem. 46 (2011) 202.

[7] C. C. C. R. de Carvalho, Res. Microbiol. 163 (2012) 125.

[8] H. Yang, Q. Tang, C. Wang, and J. Zhang, Miner. Eng. 45 (2013) 67.

[9] M. S. Kuyukina et al., J. Microbiol. Methods 46 (2001) 149.

[10] S. Lang and J. C. Philp, Antonie Van Leeuwenhoek 74 (1998) 59.

[11] J. D. Desai and I. M. Banat, Microbiol. Mol. Biol. Rev. 61 (1997) 47.

[12] N. Deo and K. A. Natarajan, Miner. Eng. 10 (1997) 1339.

[13] A. M. Raichur et al., Colloids Surf. B Biointerfaces 8 (1996) 13.

[14] S. Subramanian, D. Santhiya, and K. A. Natarajan, Int. J. Miner. Process. 72 (2003) 175.

[15] A. Vilinska and K. H. Rao, Trans. Nonferrous Met. Soc. China 18 (2008) 1403.

[16] L. M. S. de Mesquita, F. F. Lins, and M. L. Torem, Int. J. Miner. Process. 71 (2003) 31.

[17] N. Deo, K. A. Natarajan, and P. Somasundaran, Int. J. Miner. Process. 62 (2001) 27.

[18] M. Farahat et al., Miner. Eng. 21 (2008) 389.

[19] S. Pavlovic and P. R. G. Brandao, Miner. Eng. 16 (2003). 1117.

[20] S. Garip, A. C. Gozen, and F. Severcan, Food Chem. 113, 1301 (2009).

[21] H. Yang et al., Int. J. Miner. Metall. Mater. 21 (2014) 210.

E-mail: caralcaso@gmail.com 